\title{
PREVALENT NEW-USER COHORT DESIGNS FOR COMPARATIVE DRUG EFFECT STUDIES BY TIME-CONDITIONAL PROPENSITY SCORES
}

Samy Suissa, $\mathrm{PhD}$

Sophie Dell'Aniello, MSc

Erica E M Moodie, PhD

Department of Epidemiology and Biostatistics, McGill University, Montreal, Canada.

McGill Pharmacoepidemiology Research Unit, Centre for Clinical Epidemiology, Jewish General Hospital, Montreal, Canada.

Please address correspondence to:

Dr. Samy Suissa

Centre for Clinical Epidemiology, Jewish General Hospital

3755 Cote Ste-Catherine, H4.61

Montreal, Québec, Canada H3T 1E2

Tel: 514-340-7593 Fax: 514-340-7564

E-mail: samy.suissa@mcgill.ca

Words text: 4,475

Words abstract: 250

Tables: 2 Figures: 5

Running title: Prevalent new-user designs

Key words: Cohort studies; Comparative effectiveness; Database research; Drug safety; Epidemiologic design; Pharmacoepidemiology

August 16, 2016 


\section{Take-home messages}

- Real-world comparative effectiveness of drugs conducted using computerized healthcare databases very common

- Typical new-user cohort design limited to head-to-head comparisons between two new medications, using treatment-naïve patients.

- We introduce the prevalent new-user cohort design for head-to-head comparisons between a new drug and an older drug, using all patients, including the switchers from the old to the new drug.

- We introduce the notion of exposure sets to compute time-conditional propensity scores of initiating the newer drug for matching purposes.

- We used the prevalent new-user cohort design to compare the risk of heart failure with newer GLP-1 analogs to treat type 2 diabetes compared with older sulfonylureas, using all patients rather than only the treatment-naïve ones. 


\section{ABSTRACT}

Studies of the real-world comparative effectiveness of drugs conducted using computerized healthcare databases typically involve an incident new-user cohort design for head-to-head comparisons between two medications, using exclusively treatment-naïve patients. However, the desired contrast often involves one new drug compared with an older drug, of which many users of the new drug may have switched from, seriously restricting the scope of incident new-user studies.

We introduce prevalent new-user cohort designs for head-to-head comparative drug effect studies, where incident new users are scarce. We define time-based and prescription-based exposure sets to compute time-conditional propensity scores (TCPS) of initiating the newer drug and to identify matched subjects receiving the comparator drug.

We illustrate this approach using data from the United Kingdom's Clinical Practice Research Datalink (CPRD) to evaluate whether the newer GLP-1 analogs used to treat type 2 diabetes increase the risk of heart failure, in comparison with the older similarly indicated sulfonylureas. Of 170,031 users of anti-diabetic agents from 2000 onwards, 79,682 used sulfonylureas (first use 2000), while 6,196 used GLP-1 analogs (first use 2007), 75\% of which had previously used a sulfonylurea. After matching each GLP-1 analog user to a sulfonylurea user on the time-conditional propensity scores from prescription-based exposure sets, the hazard ratio of heart failure with GLP-1 use was 0.73 (95\% CI: $0.57-0.93)$.

The proposed prevalent new-user cohort design for comparative drug effects studies allows the use of all or most patients exposed to the newer drug, thus permitting a more comprehensive assessment of a new drug's safety. 


\section{INTRODUCTION}

Observational studies of the comparative effects of drugs, including effectiveness and safety, are generally based on a cohort design. ${ }^{1}$ It is recommended that cohorts be defined by new users of the drugs under study, also called incident users, to avoid missing their potential early effects and properly time the confounders. ${ }^{2,3}$ While studies of comparative effects will typically involve the head-to-head comparison of two similar drugs, one is often faced with evaluating the effect of a new drug, with no contemporaneous comparator and for which the most appropriate comparator is an older drug that has been on the market for a long time. As a result, many of the new users of the drug under study will not be treatment-naïve, but will have switched from the older comparator drug.

Although one of the inherent expectations of real-world data is that all or most users of the new drug will be included in observational studies, studies are often based strictly on treatment-naïve users who make up only a small part of the real-life user population of the new drug, particularly during the early period of its availability. An example is the study of the real life effectiveness and safety of the newer anticoagulant dabigatran used to treat atrial fibrillation (AF), where the natural comparator is warfarin, an older anticoagulant. One of the first observational studies used "anticoagulant-naïve patients", thus excluding many of the new users of dagibatran who had switched from warfarin to dabigatran, which may not fully characterize "everyday clinical practice" and the "real-world" patients desired by the study. ${ }^{4}$ It was noted that up to $50 \%$ of dabigatran users had previously used warfarin. ${ }^{5}$ Another recent example involves a cohort study comparing patients with type 2 diabetes initiating treatment with the newer class of dipeptidyl-peptidase-4 (DPP-4) inhibitors with those initiating the older sulfonylureas on the risk of pancreatic cancer, which excluded around $50 \%$ of patients with a history of sulfonylurea use prior to DPP-4 treatment. ${ }^{6}$ 
In this paper, we present approaches to design cohort studies of head-to-head comparative drug effects, particularly focussing on the situation where the comparator drug is not contemporaneous. These designs are illustrated using data from the United Kingdom's Clinical Practice Research Datalink (CPRD) to evaluate whether newer incretin-based drugs used to treat type 2 diabetes increase the risk of heart failure, in comparison with the older sulfonylureas.

\section{INCIDENT NEW-USER DESIGN}

Some studies of comparative effects will involve the comparison of two drugs of similar indication that entered the market at around the same time. Such studies can be based on new users of each of the two drugs that are naïve to the use of the other drug, subjects that we call "incident newusers". The strategy of such incident new-user comparative studies thus identifies the cohort of incident new users of the two drugs under study and makes them comparable by design or analysis. For example, a study comparing the effectiveness of two different combination inhalers in the treatment of asthma formed the cohort of all incident new users of budesonide/formoterol or fluticasone/salmeterol after a diagnosis of asthma. ${ }^{7}$ Different techniques of data analysis can then be used, such as regression or propensity scores, to match or adjust for differences in the baseline characteristics on the date of the first such prescription between the two groups.

Challenges in the design of these incident new-user comparative studies involve considerations for calendar time and prior medications. First, while the two drugs are similar in nature, they may have entered the market at somewhat different times. In such a case, it is possible that the first drug on the market was given to the more severe patients when it was the only one of the class available. ${ }^{8}$ Thus, it may be necessary to restrict the cohort entry time to a contemporaneous period when the two drugs were both available to reduce confounding issues. For the asthma study, the two inhalers 
entered the UK market in 1999 and 2001, so that cohort entry was defined only on the new users of both inhalers as of 2001. Another challenge with these studies is related to the other drugs the patients were using at the time they initiated treatment with the new drugs. Direct matching on the prior drug usage patterns may be preferable to assuming that propensity scores will inherently provide the proper balance in disease severity at the time of initiation. Another consideration that could affect confounding is whether the study drugs are initiated as replacement or as addition to the current drug regimen, which should be verified in establishing balance in disease severity at the time of initiation.

In all, incident new-user comparative cohort studies are as relatively straightforward as observational studies can be. Besides issues related to timing and co-medication patterns, common techniques of analysis to control for confounding, such as matching, propensity scores or statistical adjustment, will then provide the balanced treatment groups at cohort entry necessary to estimate comparative effects with minimal bias. ${ }^{8}$

\section{PREVALENT NEW-USER DESIGN}

A more common situation in pharmacoepidemiology involves the study of the effect of a new drug entering the market, in the absence of a direct contemporaneous comparator. For example, concerns have recently been raised regarding the safety of the newer DPP- 4 inhibitors to treat type 2 diabetes. Since these drugs are second-line treatment for type 2 diabetes, a natural comparator would be sulfonylureas, another second-line treatment, though already in use for a very long time. The particularity of this situation is that many of the new users of the newer DPP-4 inhibitors were previously on sulfonylureas, the comparator; they were thus switched from one second-line treatment to another. An incident new-user analysis could be performed by comparing incident new users of 
incretin-based drugs with incident new users of sulfonylureas, including only patients who were treatment-naïve to both drugs. This, however, would exclude the large number of subjects who switched from sulfonylureas to the DPP-4 inhibitors, which could represent a significant number of patients and clinically relevant subset. In such instances, a prevalent new-user comparative cohort approach could be used.

Initially, the base cohort from which the comparative study cohorts will be identified must be formed from all users of the comparator drug and of the drug under study. This base cohort will thus identify all users of the study drug who had used the comparator and switched, as well as those who directly initiated the study drug, also providing the pool from which the comparator drugs users will be selected. Figure 1 depicts a base cohort formed of all subjects who initiated the comparator drug, some switching to the study drug, the others continuing on the comparator, as well as the subjects who initiated the study drug without ever using the comparator. The main challenge with the design of prevalent new-user comparative cohort studies is the selection of comparator drug users for the subjects who switched from the comparator drug to the study drug.

We present two ways this can be achieved. We first define the exposure set as the set of subjects in the base cohort exposed to the comparator drug at the point that a subject switched to the study drug. The point of switching can be demarcated in two ways, by the time from the first prescription of the comparator drug up to the point of switching, or the number of prescriptions of the comparator drug received up to the point of switching.

\section{Time-based exposure sets}

If time from the first prescription of the comparator drug is deemed important in measuring the disease course, time-based exposure sets can be used to determine the point at which 
comparability will be established. This corresponds to the same time axis used to define the base cohort, namely the time since the first comparator prescription, as depicted in Figure 1. It is obvious from this picture that identifying comparator prescriptions given at the same time as the point at which the patients switched to the study drug is nearly impossible because of the granularity of the time scale. Thus, time-based exposure sets must be defined with a time interval, such as \pm 1 month, where all patients with a comparator prescription in that time span are said to belong to the exposure set and the set is defined by each patient's prescription date. Figure 2 shows the time-based exposure sets from the base cohort. They provide equivalent time points in the disease course at which confounder patient characteristics can be measured. While this approach does not provide an elegant vertical line on the time axis, it allows for a sufficiently accurate account of time since entry into the base cohort.

\section{Prescription-based exposure sets}

If the number of comparator prescriptions rather than the time since the first comparator prescription is deemed important in quantifying the disease progress, prescription-based exposure sets can be used to determine the point of comparability. Figure 3 depicts a base cohort defined by the prescription number and shows the corresponding prescription-based exposure sets defined by each subject who switched to the study drug. Prescription-based exposure sets do not consider the time between prescriptions.

\section{Time-conditional propensity scores}

To identify the comparator drug user most similar to the patients who switched to the study drug, time-conditional propensity scores (TCPS) can be used. They are named time-conditional 
because they depend on the time-varying patient characteristics measured at the point of the prescription-based or time-based exposure sets and the positivity assumption is verified conditionally within each exposure set. To compute the propensity of switching to the study drug, versus continuing on the comparator drug, as a function of the time-varying patient characteristics measured at the point of the exposure set, the time-dependent Cox proportional hazards model, or equivalently conditional logistic regression, can be used to conserve the matching induced by the exposure set.$^{9}$ The resulting estimated model is used to compute the time-conditional propensity score within each exposure set to identify the subject in the exposure set with the closest value to that of the switcher, thus identifying their matched comparator. For the purposes of the positivity assumption, the time-conditional propensity score of the switcher should lie within the range of the time-conditional propensity scores of the members of the corresponding exposure set.

To emulate the randomized trial, this process can be initiated with the first chronological index study drug subject and repeated sequentially. Additionally, once a patient has been selected into the comparator group, they are not considered any longer in subsequent exposure sets as potential comparators. Thus, each subject who initiated the study drug will have a comparator user, matched on the time-conditional propensity score. Cohort entry is taken as the date of the first prescription of the study drug use and the corresponding date for the matched comparator drug user, with follow-up depending on the question under study. Figure 4 depicts a study cohort selected from the prescription-based exposure sets of Figure 2. The Appendix provides a step-by-step description for the design of a prevalent new-user cohort using prescription-based exposure sets.

A potential challenge in computing the time-conditional propensity scores is the size of the exposure sets which can make the time-varying Cox modeling technically unmanageable. For example, a study of the safety of incretin-based drugs using the CPRD, versus sulfonylureas as the comparator, 
could very well involve a base cohort of over 100,000 users of older antidiabetic drugs with close to 4,000 switchers to incretin-based drugs. ${ }^{10}$ Such an analysis would involve 4,000 exposure sets with numbers of subjects in each set as high as 100,000 , clearly technically infeasible with the timedependent Cox model, particularly if the number of potential confounders is large and involves multiple strata. One approach is to select random samples of 10, 20 or 100 prescriptions from each exposure set to estimate the time-conditional propensity scores of switching to the study drug as a function of the patients characteristics at the point of the exposure set. These can be computed using conditional logistic regression, matched on the exposure set, with the resulting relative odds accurately estimating the corresponding relative hazards. The computed propensity odds score for the index switcher is used to identify the corresponding matched patient as the subject with the closest value from all members of the exposure set, not only the sampled ones.

\section{AVOIDING SELECTION BIAS}

Most cohort studies involve exclusion criteria that define a homogenous population. These criteria usually represent a history of clinical conditions, including a history of the outcome event of interest. For instance, the study of whether incretin-based drugs are associated with pancreatic cancer will necessarily involve a cohort free of this cancer at cohort entry. Applying this exclusion is rather straightforward for the incident new-user comparative cohorts, with attention given to a baseline period of equal duration for the two drug groups under study, to avoid potential differential exclusions that may be associated with the outcome.

For the prevalent new-user comparative cohort design, however, the potential for such selection bias from exclusion criteria is high. While the exclusion of subjects who have a history of the event under study prior to cohort entry is straightforward for the incident new users in both groups, it 
is more subtle for the prevalent users. Without a careful systematic approach, one could easily end up excluding outcome events, not only history events, or misclassifying history events as outcome events. This was observed in a cohort study of the effect of metformin on cancer incidence. ${ }^{11}$ Using a population of diabetics, all 4,085 new users of metformin between 1994 and 2003 were individually matched with 4,085 non-users on year of diabetes diagnosis, with each pair assigned the cohort entry date as the date of the first metformin prescription of the user. In choosing comparators, however, a potential matched non-user who had a diagnosis of cancer prior to the index date was discarded, but "was potentially available for a different metformin user...this process was repeated until suitable comparators were identified". The net effect of such selection of non-users with replacement is that the non-user group will have zero subjects excluded because of cancer prior to the cohort entry and what should have been "prior" cancers subject to exclusion were transferred to the follow-up period and counted as outcome events, thus artificially increasing the rate of cancer in the non-user comparator group. ${ }^{12}$

To ensure that such selection bias is avoided, it is essential to perform the identification of matched comparator subjects in a systematic and chronological manner. First, this should be done blinded to the occurrence or timing of outcome events in the base cohort. Second, after propensity scores have been computed, identification of matched comparators should be conducted in chronological order, with the first new user of the study drug in calendar time matched first to a comparator, the second next, and so on. At this point, if the new user of study drug has a history of the exclusion event, they must be excluded outright from any further selection into the comparative cohort analysis. If the new user is eligible, a comparator with the closest propensity score is selected. If this comparator has a history of the exclusion event, they must be excluded from any further selection and the next closest match without such a history can be used to match to the new user. The chronological 
approach to selecting comparators will thus ensure that proper outcome events are not inappropriately excluded and exclusion events are not incorrectly counted as outcomes. It is also important not to initially exclude all new users of the study drug with a history of the outcome event, since some of these subjects may be eligible as comparator exposures prior to switching.

A second issue relates to the comparators that, during follow-up, switch from the old comparator drug to the new drug. Two choices are possible. The first is to censor the comparator follow-up at the point of switch, then include the subject as a new-user of the new drug from this point onwards, while identifying a matched comparator at that point. The second is to not re-use these subjects if they were selected as comparators, while leaving the option open to censor or not the follow-up at the point of switch.

\section{ILLUSTRATION}

To illustrate the method, we use data from the United Kingdom's Clinical Practice Research Datalink (CPRD) to study whether the incretin-based glucagon-like peptide-1 receptor agonists (GLP-1 analogs) used to treat type 2 diabetes increase the risk of heart failure. ${ }^{10}$ These drugs work in the same pathway but are considered more potent than DPP-4 inhibitors that have been reported to be associated with an increase in the risk of heart failure. ${ }^{13}$ These second-line drugs, that entered the UK market in 2007, were compared with sulfonylureas, another second-line treatment for type 2 diabetes which has been around for decades, thus requiring a prevalent new-user comparative cohort design.

We used data from a previous study to identify the base cohort of 170,031 users of antidiabetic agents between 2000 and 2013, which included 6,196 users of GLP-1 analogs and 79,682 users of sulfonylureas. ${ }^{14}$ The first prescription for a GLP-1 analog was in July 2007 while sulfonylureas were used since January 2000. Of the 6,196 users of GLP-1 analogs, close to $75 \%$ had previously used a 
sulfonylurea, the comparator drug. Thus, a purely incident new-user cohort study would only be based on 1,633 new users of this newer agent rather than all 6,196 new users. The prevalent new-user comparative cohort approach was thus used with time-based exposure sets defined by the duration of treated diabetes and prescription-based exposure sets by the number of prescriptions of sulfonylurea prior to initiating the GLP-1 analog. The time-conditional propensity scores of initiating GLP-1 analog use relative to sulfonylurea use were estimated using conditional logistic regression, after either timebased or prescription-based matching. We present two analyses, namely re-using the selected sulfonylurea comparators who subsequently switched to GLP-1 analogs in the group exposed to GLP-1 analogs and not re-using them. In all analyses, follow-up was censored at the point of switch for the comparators.

Figure 5a displays the time-conditional propensity score distribution, highlighting the different profiles for the new users of GLP-1 analogs compared with users of sulfonylureas, while Figure $5 \mathrm{~b}$ depicts it after prescription-based time-conditional propensity score matching. Table 1 describes the cohorts formed by pairing the new users of a GLP-1 analog with a corresponding user of sulfonylurea within the same exposure set and calendar year, unmatched and matched on the time-conditional propensity scores computed using prescription-based and time-based exposure sets. In both matched cohorts, the mean difference in propensity score across matched set was below 0.002 . The prescription-based approach that matched on the number of prior prescriptions of sulfonylurea (mean 21.7) resulted in balanced duration of treated diabetes (5.2 years). On the other hand, the time-based approach that matched on the duration of treated diabetes (5.2 years) resulted in an imbalance in the number of prior prescriptions of sulfonylurea (means 21.8 versus 19.9) and the proportion of sulfonylurea users (73.6\% versus $94.0 \%)$. 
Table 2 shows that the prescription-based and time-based approaches produce similar results. For the tighter matched prescription-based approach, the hazard ratio of heart failure associated with GLP-1 analog use was 0.73 (95\% CI: 0.57-0.93) when all exposed subjects were included and somewhat lower at 0.67 (95\% CI: 0.52-0.88) when these subjects were not re-used. Among the incident new-users, which included $26 \%$ of the subjects, the latter hazard ratio was 0.59 (95\% CI: 0.33 1.07), while among the prevalent new-users, it was 0.71 (95\% CI: 0.52-0.96). The recent LEADER randomized trial of a GLP-1 analogue did not find an excess risk of heart failure (HR 0.87; 95\% CI: $0.73-1.05) \cdot{ }^{15}$

\section{DISCUSSION}

We introduced designs for new user cohort studies of comparative drug effects, distinguishing between incident new-user and prevalent new-user designs, particularly focussing on the latter where most new users of the study drug were previously using the comparator. In this very common situation, where a recently marketed drug is to be compared to an older established alternative, we proposed prevalent new-user comparative cohort designs that use time-based and prescription-based exposure sets to compute time-conditional propensity scores of initiating the new drug as a tool to identify matched subjects using the comparator drug at the same point in the course of disease. We also showed how the time-conditional propensity scores could be estimated using sampling within the exposure sets when the cohorts are too large.

While time-dependent propensity scores have been used in pharmacoepidemiology, these were not developed for the specific context of the prevalent new-user design we are addressing. ${ }^{16,17}$ In particular, these used standard logistic regression, which does not account for the entire history of use of the comparator drug in a prevalent cohort. As such, our use of the time-dependent Cox proportional 
hazards model, or its equivalent conditional logistic regression model, to estimate the time-conditional propensity scores over time is distinctive. The label "time-conditional" is added to the term propensity score to remind that its computation is done by updating the time-varying covariates at each exposure set, and that matching is done within the exposure sets, including the verification of the positivity assumption which should be done within each exposure set.

Several methodological issues need to be considered with these designs. First, one could distinguish between patients who switched from the old (comparator) drug to the newer one from those who added the newer drug. The latter could indicate more severe disease so that careful assessment of confounding should be entertained, focussing on recent clinical data prior to adding the newer drug. Second, there could be effect-modification by history of prior use of the comparator drug, which would necessitate distinguishing between the incident and prevalent cohorts. For instance, because the prevalent users could have tolerated or survived the period of early harm, the smaller cohort of incident users may be necessary to also assess the impact of the drug at the point of initiation. Conversely, in a study of the long-term effects of insulin glargine on the risk of breast cancer, a prevalent new-user comparative cohort design was used to match the users of insulin glargine with users of other insulins. ${ }^{18}$ The risk of breast cancer increased after 5 years of insulin glargine use among the prevalent new-users who had been on another insulin prior to starting glargine (HR: 2.7; 95\% CI: 1.1-6.5), but not among the incident, insulin-naïve, new users. Nevertheless, the overall effect estimated by the design provides the marginal effect had everyone who could receive the new treatment received it - regardless of whether those patients were treatment naïve - relative to the risk of the outcome had all patients been prescribed the old treatment, analogous to an average treatment effect on the treated. 
We presented two options in selecting the comparator group, either "re-using" or not as exposed the comparators who, during follow-up, switch from the old comparator drug to the newer drug. A third option that should be avoided is to select the comparators among those who never switch to the newer drug. This option, that is based on "looking in the future" can lead to immortal time bias and should thus be precluded. However, this bias will be trivial if the number of users of the newer drug is small compared to the users of the comparator in the base cohort.

We presented two types of exposure sets, time-based and prescription-based. In our illustration, the time-based approach led to somewhat unequal matching on the number of prior prescriptions. Since it represents the basis for the comparator exposure, one could deem that the prescription-based exposure set approach is preferable. Indeed, to emulate the randomized trial, the principle is to identify, at the physician visit that led to the patient being switched from the comparator drug to the study drug, a comparable patient who has the same history of comparator drug use and of other characteristics, but who on that visit continued on the comparator drug. The prescription-based exposure sets thus provide equivalent points in the disease course in terms of comparator drug history and equivalent points at which confounder patient characteristics can be measured. Nevertheless, if disease duration is also deemed to be important, a combination of time-based and prescription-based exposure sets is certainly feasible.

It is noteworthy that many pharmacoepidemiologic studies do not have a specific comparator drug but rather aim to compare the study drug to "non-use". In these studies, while the timing of the first prescription of the study drug is directly measurable, selecting the time point for non-use is challenging. Our approach can also be used simply by using a prescription for any drug other than the study drug as the comparator. 
Further research is needed to understand the limits of prevalent new-user comparative cohort designs. First, we are uncertain about the impact of "re-using" or not the comparators, in particular the potential impact of non-independence in the estimation of confidence intervals, which could require techniques based on bootstrap sampling. Second, if we select not to re-use the comparator subjects who switch to the newer drug, it remains uncertain whether and in which situation to censor the follow-up at the point of switch. Third, one must be conscious about adjustment for factors in the causal pathway when computing the propensity score, ${ }^{3}$ although factors that could be affected by the old comparator drug, such as the level of HbA1c, would have to be considered differently for the prevalent and incident new-users. In this case, propensity scores that also include an interaction term between the incident/prevalent status and this factor could be considered. Fourth, bias from prescription-based exposure sets that do and do not require a physician visit for the matching prescriptions could be investigated. Some of these issues will certainly also need to be considered in the contexts of whether the outcome under study is an acute event, such as an exacerbation or a stroke, which occurs during exposure, or a long-term event such as cancer or osteoporotic fracture, which can occur long after exposure. Another issue is that the prescriptionbased approach may become computationally cumbersome in chronic conditions where the cohort is large and the prior number of prescriptions of the comparator drug is large and highly variable. In this case, simplifications could be made by matching on the number of prescriptions of the comparator drug use in the prior year or two, for example, rather than the entire history. Statistical adjustment for the use of this comparator drug previous to this prior year could then be used to supplement the design.

In all, studies of comparative effectiveness of drugs are becoming more widespread, particularly with the influx of new computerized healthcare databases. The traditional incident new- 
user comparative cohort design, exclusively based on treatment-naïve subjects, is a desirable approach for head-to-head comparisons between two medications, but often seriously restricts the size of studies, with calls for ways to incorporate prevalent users in epidemiologic studies. ${ }^{19}$ Our proposed prevalent new-user comparative cohort designs allow the use of all or most patients exposed to the newer drug, thus providing a more complete assessment of a new drug's safety.

\section{ACKNOWLEDGMENTS}

This research was funded in part by a grant from the Canadian Institutes of Health Research (CIHR) and the Canadian Foundation for Innovation (CFI). S Suissa is the recipient of the James McGill Chair award. 


\section{References}

1. Cox E, Martin BC, van ST, Garbe E, Siebert U, Johnson ML. Good research practices for comparative effectiveness research: approaches to mitigate bias and confounding in the design of nonrandomized studies of treatment effects using secondary data sources: the International Society for Pharmacoeconomics and Outcomes Research Good Research Practices for Retrospective Database Analysis Task Force Report--Part II. Value Health 2009;12(8):10531061.

2. Ray WA. Evaluating medication effects outside of clinical trials: new-user designs. Am J Epidemiol 2003;158(9):915-920.

3. Johnson ES, Bartman BA, Briesacher BA et al. The incident user design in comparative effectiveness research. Pharmacoepidemiol Drug Saf 2013;22(1):1-6.

4. Larsen TB, Rasmussen LH, Skjoth F et al. Efficacy and safety of dabigatran etexilate and warfarin in "real-world" patients with atrial fibrillation: a prospective nationwide cohort study. J Am Coll Cardiol 2013;61(22):2264-2273.

5. Sorensen R, Gislason G, Torp-Pedersen C et al. Dabigatran use in Danish atrial fibrillation patients in 2011: a nationwide study. BMJ Open 2013;3(5).

6. Gokhale M, Buse JB, Gray CL, Pate V, Marquis MA, Sturmer T. Dipeptidyl-peptidase-4 inhibitors and pancreatic cancer: a cohort study. Diabetes Obes Metab 2014;16(12):1247-1256.

7. Suissa S, Dell'Aniello S, Ernst P. Effectiveness of combination therapies in asthma: an observational study. Pulm Pharmacol Ther 2009;22(3):194-198.

8. Gagne JJ, Bykov K, Willke RJ, Kahler KH, Subedi P, Schneeweiss S. Treatment dynamics of newly marketed drugs and implications for comparative effectiveness research. Value Health 2013;16(6):1054-1062.

9. Lu B. Propensity score matching with time-dependent covariates. Biometrics 2005;61(3):721728.

10. Yu OH, Filion KB, Azoulay L, Patenaude V, Majdan A, Suissa S. Incretin-based drugs and the risk of congestive heart failure. Diabetes Care 2015;38(2):277-284.

11. Libby G, Donnelly LA, Donnan PT, Alessi DR, Morris AD, Evans JM. New users of metformin are at low risk of incident cancer: a cohort study among people with type 2 diabetes. Diabetes Care 2009;32(9):1620-1625.

12. Suissa S, Azoulay L. Metformin and the risk of cancer: time-related biases in observational studies. Diabetes Care 2012;35(12):2665-2673.

13. Filion KB, Suissa S. DPP-4 Inhibitors and Heart Failure: Some Reassurance, Some Uncertainty. Diabetes Care 2016;39(5):735-737. 
14. Filion KB, Azoulay L, Platt RW et al. A Multicenter Observational Study of Incretin-based Drugs and Heart Failure. N Engl J Med 2016;374(12):1145-1154.

15. Marso SP, Daniels GH, Brown-Frandsen K et al. Liraglutide and Cardiovascular Outcomes in Type 2 Diabetes. N Engl J Med 2016.

16. Ray WA, Liu Q, Shepherd BE. Performance of time-dependent propensity scores: a pharmacoepidemiology case study. Pharmacoepidemiol Drug Saf 2015;24(1):98-106.

17. Sanni AM, Groenwold RH, Pestman WR et al. Time-dependent propensity score and colliderstratification bias: an example of beta2-agonist use and the risk of coronary heart disease. Eur J Epidemiol 2013;28(4):291-299.

18. Suissa S, Azoulay L, Dell'Aniello S, Evans M, Vora J, Pollak M. Long-term effects of insulin glargine on the risk of breast cancer. Diabetologia 2011;54(9):2254-2262.

19. Vandenbroucke J, Pearce N. Point: incident exposures, prevalent exposures, and causal inference: does limiting studies to persons who are followed from first exposure onward damage epidemiology? Am J Epidemiol 2015;182(10):826-833. 


\section{Table 1}

Baseline characteristics for prevalent new-user comparative cohorts of type 2 diabetes patients pairing, using prescription-based and time-based exposure sets, the new users of a GLP-1 analog with a corresponding user of a sulfonylurea within the same exposure set and calendar year, unmatched and matched on the time-conditional propensity score

\begin{tabular}{|c|c|c|c|c|c|c|}
\hline & \multicolumn{3}{|c|}{ Prescription-based cohort } & \multicolumn{3}{|c|}{ Time-based cohort } \\
\hline & GLP-1 analog & $\begin{array}{c}\text { Unmatched } \\
\text { Sulfonylurea }\end{array}$ & $\begin{array}{c}\text { Matched } \\
\text { Sulfonylurea } \\
\end{array}$ & GLP-1 analog & $\begin{array}{c}\text { Unmatched } \\
\text { Sulfonylurea }\end{array}$ & $\begin{array}{c}\text { Matched } \\
\text { Sulfonylurea } \\
\end{array}$ \\
\hline Number of subjects & 6,195 & 6,195 & 6,195 & 6,196 & 6,196 & 6,196 \\
\hline Age at cohort entry, mean \pm sd & $56.0 \pm 10.5$ & $65.8 \pm 12.9$ & $56.4 \pm 11.4$ & $56.0 \pm 10.4$ & $66.9 \pm 12.4$ & $56.5 \pm 11.4$ \\
\hline Female sex, \% & 45.1 & 40.2 & 44.1 & 45.1 & 40.1 & 44.4 \\
\hline \multicolumn{7}{|l|}{ Year of cohort entry, \% } \\
\hline 2007 & 1.1 & 1.1 & 1.1 & 1.1 & 1.1 & 1.1 \\
\hline 2008 & 6.1 & 6.1 & 6.1 & 6.1 & 6.1 & 6.1 \\
\hline 2009 & 13.9 & 13.9 & 13.9 & 13.9 & 13.9 & 13.9 \\
\hline 2010 & 18.7 & 18.7 & 18.7 & 18.8 & 18.8 & 18.8 \\
\hline 2011 & 18.0 & 18.0 & 18.0 & 18.0 & 18.0 & 18.0 \\
\hline 2012 & 19.8 & 19.8 & 19.8 & 19.8 & 19.8 & 19.8 \\
\hline 2013 & 15.9 & 15.9 & 15.9 & 15.9 & 15.9 & 15.9 \\
\hline 2014 & 6.4 & 6.4 & 6.4 & 6.4 & 6.4 & 6.4 \\
\hline Smoker, \% & 57.5 & 54.4 & 58.6 & 57.5 & 52.9 & 57.5 \\
\hline Obese, $\%$ & 93.2 & 52.9 & 93.7 & 93.2 & 51.8 & 94.0 \\
\hline Alcohol Abuse, \% & 6.3 & 6.2 & 6.2 & 6.3 & 6.2 & 6.6 \\
\hline Duration of treated diabetes, years, mean \pm sd & $5.2 \pm 3.0$ & $4.0 \pm 3.1$ & $5.2 \pm 3.1$ & $5.2 \pm 3.0$ & $5.2 \pm 3.0$ & $5.2 \pm 3.0$ \\
\hline Prior sulfonylurea prescription, $\%$ & 73.6 & 73.6 & 73.6 & 73.6 & 96.5 & 94.1 \\
\hline Prior sulfonylurea prescriptions, mean \pm sd & $21.7 \pm 26.5$ & $21.7 \pm 26.5$ & $21.7 \pm 26.5$ & $21.8 \pm 27.6$ & $36 \pm 36.3$ & $19.9 \pm 21.4$ \\
\hline Prior insulin use, $\%$ & 14.5 & 3.7 & 12.8 & 14.5 & 5.0 & 12.1 \\
\hline
\end{tabular}




\begin{tabular}{|c|c|c|c|c|c|c|}
\hline Prior metformin use, \% & 91.4 & 77.6 & 90.4 & 91.4 & 76.0 & 90.9 \\
\hline \multicolumn{7}{|l|}{ Hemoglobin A1c, \% } \\
\hline$\leq 7 \%[\leq 53 \mathrm{mmol} / \mathrm{mol}]$ & 6.7 & 28.9 & 5.2 & 6.7 & 37.0 & 5.9 \\
\hline $7.1-8.0 \%[54-64 \mathrm{mmol} / \mathrm{mol}]$ & 15.6 & 29.7 & 13.9 & 15.6 & 30.7 & 15.2 \\
\hline$>8 \%[>64 \mathrm{mmol} / \mathrm{mol}]$ & 77.0 & 38.6 & 80.2 & 77.0 & 31.4 & 78.0 \\
\hline Missing & 0.7 & 2.8 & 0.7 & 0.7 & 0.9 & 0.9 \\
\hline \multicolumn{7}{|c|}{ Comorbidity in year prior to cohort entry, $\%$} \\
\hline Heart failure & 4.6 & 6.8 & 4.8 & 4.6 & 6.6 & 5.0 \\
\hline Hypertension & 63.6 & 63.5 & 63.8 & 63.6 & 65.6 & 63.5 \\
\hline Coronary/ischemic heart arterial & 23.2 & 28.4 & 23.1 & 23.2 & 29.4 & 23.0 \\
\hline Atrial fibrillation & 4.6 & 9.4 & 4.8 & 4.6 & 9.5 & 4.4 \\
\hline Hypercholesterolemia & 28.8 & 28.0 & 28.3 & 28.8 & 29.9 & 29.2 \\
\hline Stroke & 3.1 & 6.3 & 3.1 & 3.1 & 6.2 & 3.2 \\
\hline Peripheral arterial/venous disease & 3.8 & 6.1 & 3.9 & 3.8 & 6.2 & 3.8 \\
\hline Acute or chronic pancreatitis & 0.9 & 2.1 & 0.9 & 0.9 & 1.7 & 0.9 \\
\hline Neuropathy & 20.5 & 17.9 & 19.4 & 20.5 & 20.1 & 19.7 \\
\hline Renal disease & 13.9 & 25.1 & 13.8 & 13.9 & 25.6 & 14.3 \\
\hline Retinopathy & 37.7 & 34.9 & 37.5 & 37.7 & 42.2 & 37.4 \\
\hline Cancer & 5.8 & 11.9 & 6.6 & 5.8 & 11.7 & 5.9 \\
\hline \multicolumn{7}{|c|}{ Medications in year prior to cohort entry, $\%$} \\
\hline ACE inhibitors & 51.6 & 48.9 & 51.3 & 51.5 & 50.7 & 51.0 \\
\hline Angiotensin receptor blockers & 20.8 & 16.9 & 20.5 & 20.8 & 18.3 & 20.1 \\
\hline Beta-blockers & 24.4 & 26.7 & 24.3 & 24.5) & 27.0 & 24.1 \\
\hline Calcium-channel blockers & 29.9 & 31.6 & 29.8 & 29.9 & 33.1 & 29.7 \\
\hline Diuretics & 35.7 & 36.5 & 35.2 & 35.7 & 36.9 & 35.8 \\
\hline Antiarrhythmic & 3.4 & 4.5 & 3.5 & 3.4 & 4.5 & 3.3 \\
\hline Cardiac glycosides & 1.9 & 5.1 & 1.8 & 1.9 & 4.8 & 1.8 \\
\hline
\end{tabular}

22 


\begin{tabular}{lcccccc}
\hline Nitrates & 5.6 & 7.2 & 5.6 & 5.6 & 7.2 & 5.4 \\
Statins & 81.9 & 77.6 & 81.6 & 81.9 & 80.0 & 81.1 \\
Other lipid lowering drugs & 6.8 & 4.4 & 6.3 & 6.8 & 4.7 \\
\hline
\end{tabular}


Table 2

Crude and adjusted hazard ratios of heart failure associated with GLP-1 analog exposure compared with sulfonylurea in patients with type 2 diabetes, using the prevalent new-user comparative cohorts identified from prescription-based and time-based matching, that include or do not include the censored GLP-1 exposed subjects

\begin{tabular}{|c|c|c|c|c|c|c|}
\hline & $\begin{array}{c}\text { Number of } \\
\text { patients }\end{array}$ & $\begin{array}{l}\text { Number } \\
\text { of events }\end{array}$ & $\begin{array}{l}\text { Person- } \\
\text { years }\end{array}$ & $\begin{array}{c}\text { Rate per } \\
1000 \text { per } \\
\text { year }\end{array}$ & $\begin{array}{c}\text { Crude* } \\
\text { HR }\end{array}$ & $\begin{array}{l}\text { Adjusted† HR } \\
(95 \% \mathrm{CI})\end{array}$ \\
\hline \multicolumn{7}{|c|}{$\begin{array}{l}\text { Prescription-based cohort approach } \\
\text { Including censored GLP-1 exposed subjects }\end{array}$} \\
\hline Sulfonylurea & 6,195 & 139 & 13,018 & 10.7 & 1.0 & 1.0 (Reference) \\
\hline GLP-1 analog & 6,195 & 122 & 15,786 & 7.7 & 0.73 & $0.73(0.57-0.93)$ \\
\hline \multicolumn{7}{|c|}{ Not Including censored GLP-1 exposed subjects } \\
\hline Sulfonylurea & 5,296 & 131 & 11,788 & 11.1 & 1.0 & 1.0 (Reference) \\
\hline GLP-1 analog & 5,296 & 108 & 14,009 & 7.7 & 0.70 & $0.67(0.52-0.88)$ \\
\hline \multicolumn{7}{|c|}{$\begin{array}{l}\text { Time-based cohort approach } \\
\text { Including censored GLP-1 exposed subjects }\end{array}$} \\
\hline Sulfonylurea & 6,196 & 135 & 13,088 & 10.3 & 1.0 & 1.0 (Reference) \\
\hline GLP-1 analog & 6,196 & 122 & 15,790 & 7.7 & 0.76 & $0.74(0.58-0.95)$ \\
\hline \multicolumn{7}{|c|}{ Not Including censored GLP-1 exposed subjects } \\
\hline Sulfonylurea & 5,333 & 123 & 11,859 & 10.4 & 1.0 & 1.0 (Reference) \\
\hline GLP-1 analog & 5,333 & 108 & 14,087 & 7.7 & 0.75 & $0.72(0.55-0.95)$ \\
\hline
\end{tabular}

* Crude, after matching on conditional propensity scores

† After matching on time-conditional propensity scores, and adjusted further for the decile of propensity score and variables in Table 1. 
Figure 1

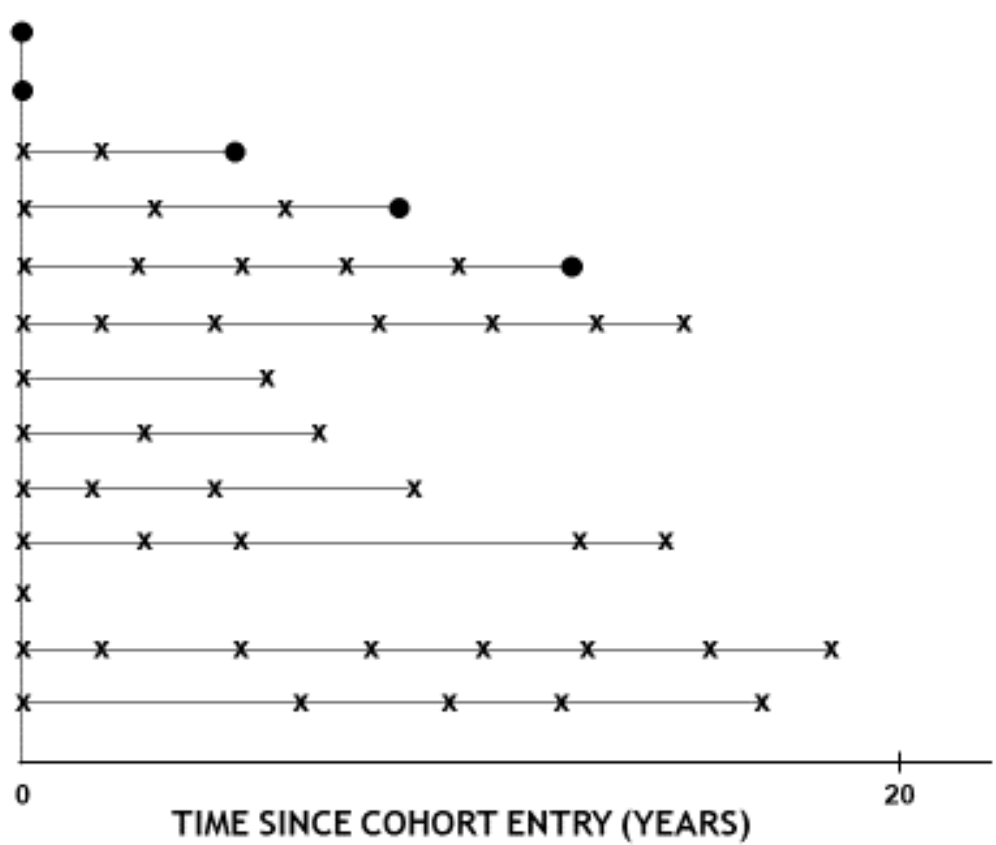


Figure 2

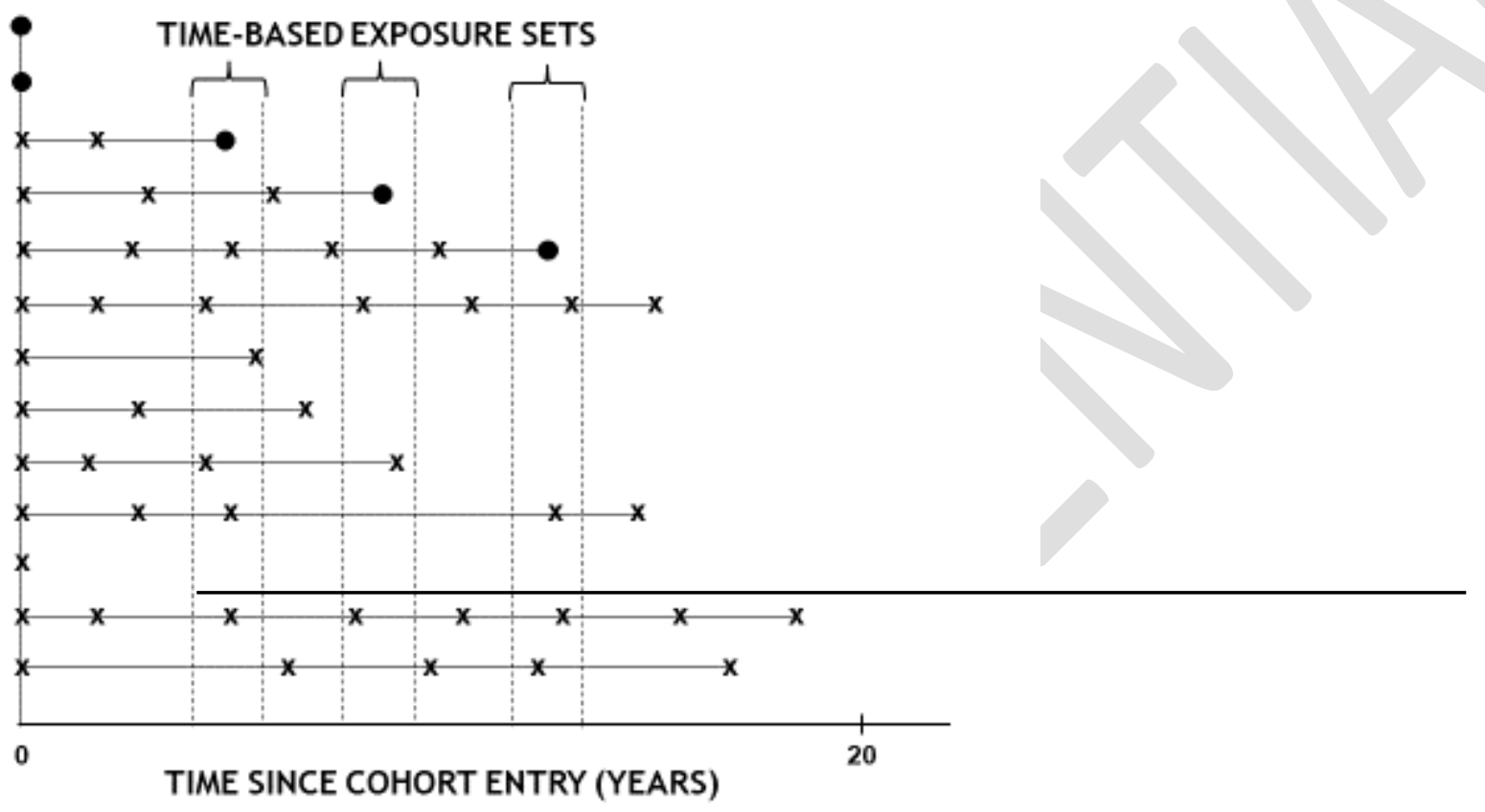


Figure 3

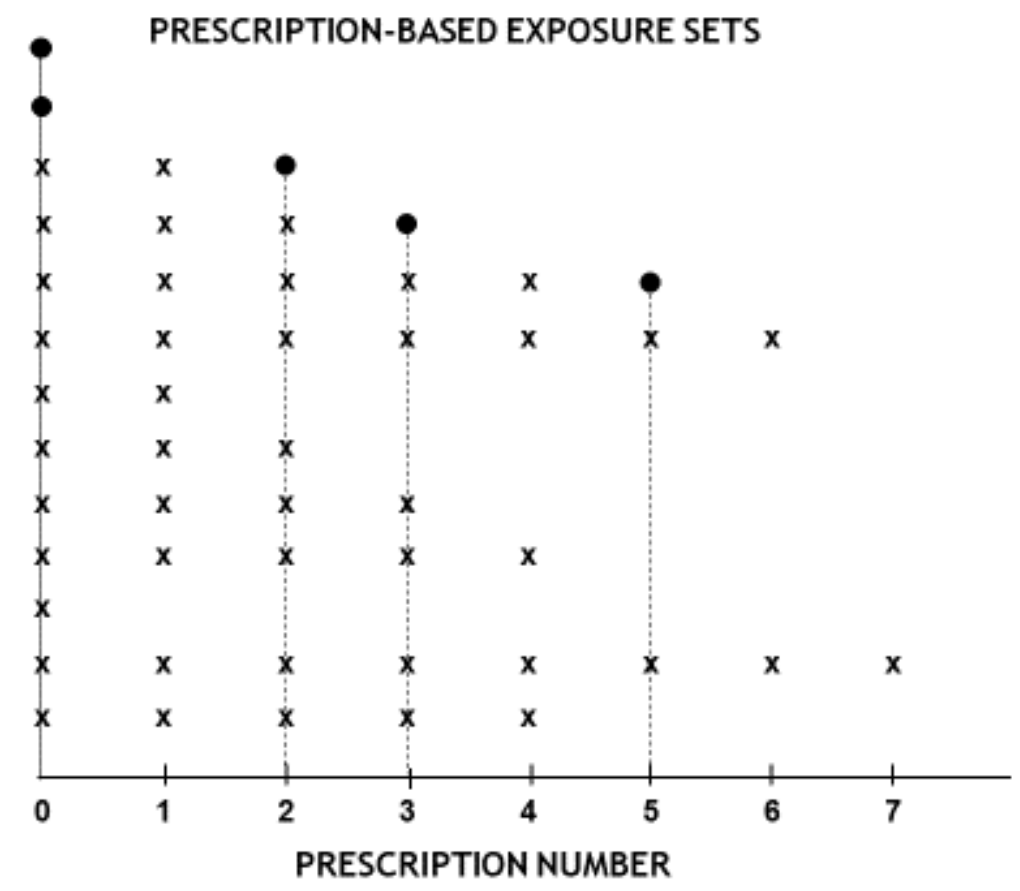


Figure 4

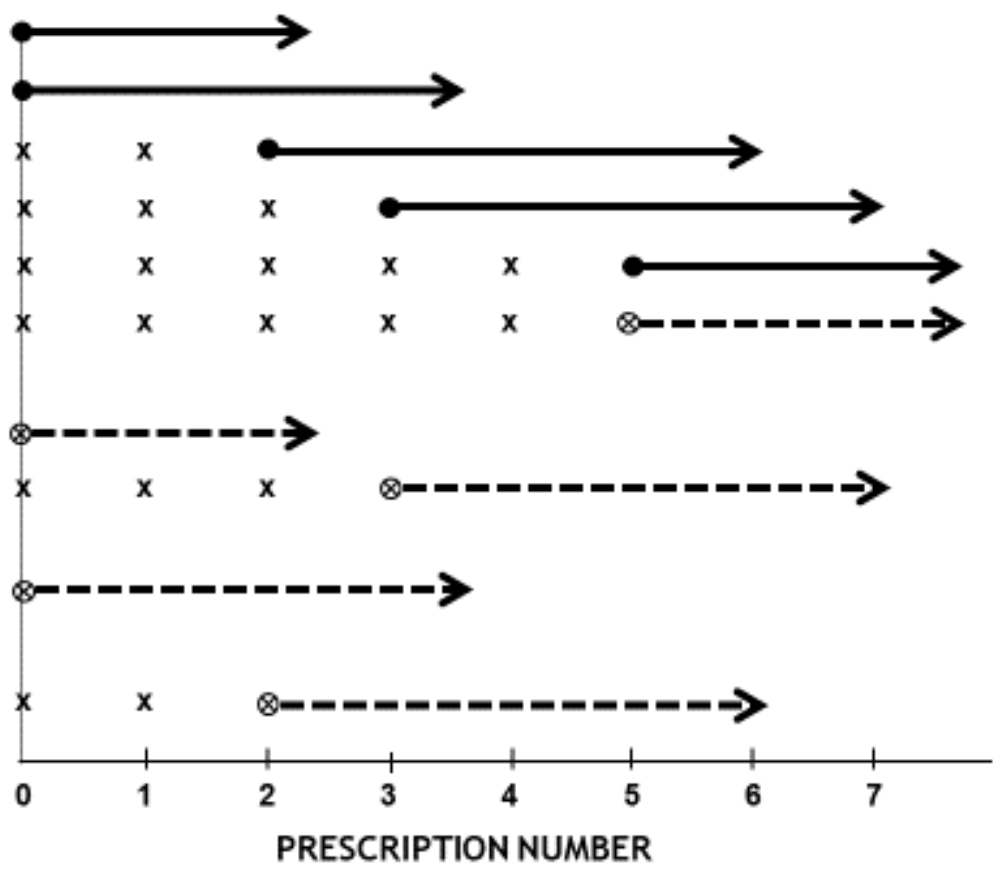


Figure 5a

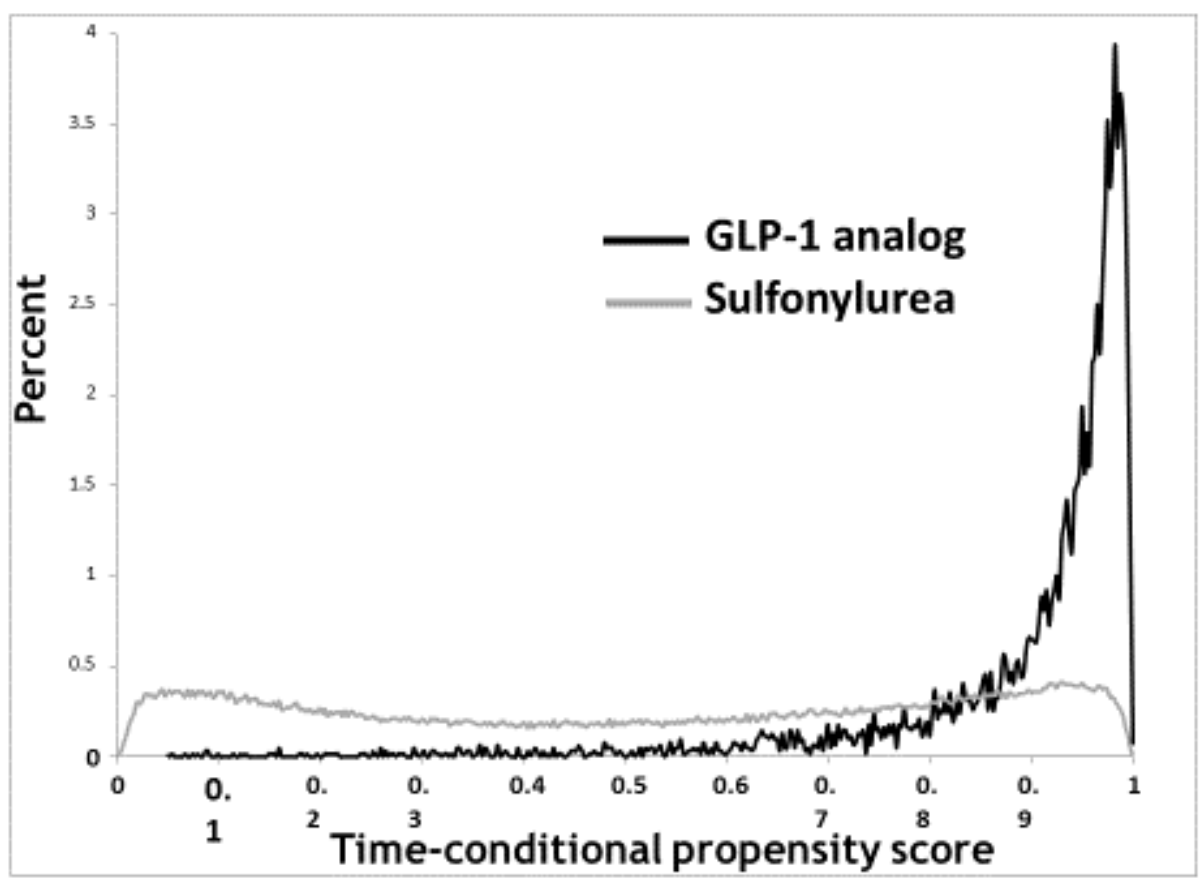


Figure $5 b$

30 

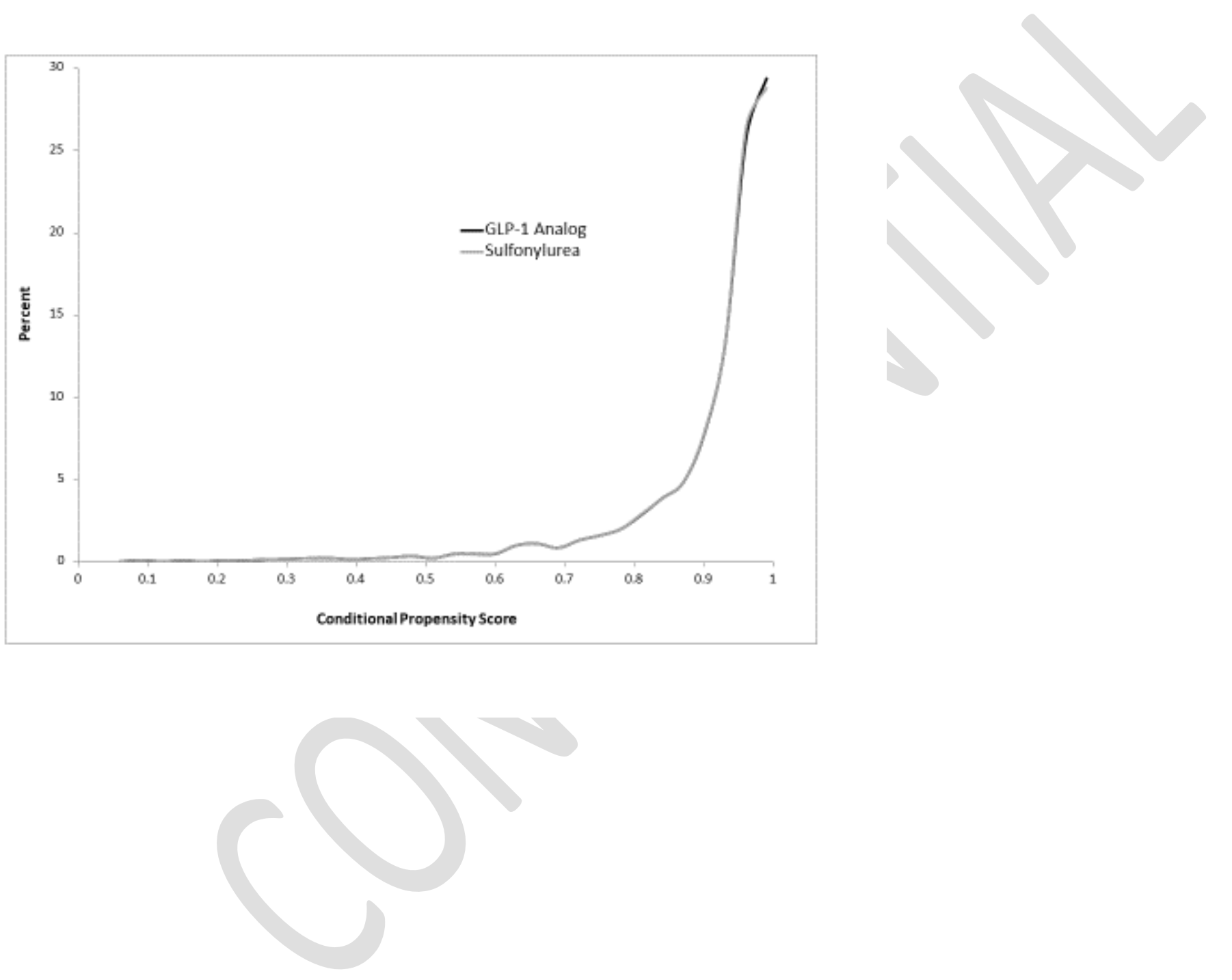


\section{Appendix}

\section{Step-by-step algorithm for the design of a prevalent new-user cohort using prescription- based exposure sets}

1. Form the base cohort of all users of the drug under study and the comparator drug.

2. Form the prescription-based exposure sets: For every new user of the study drug, identify from the base cohort every subject who has at least the same number of prior prescriptions of the comparator drug and identify the corresponding matching prescription number

3. Perform a single Cox proportional hazards regression or conditional logistic regression analysis using all the exposure sets to derive the time-conditional propensity scores including all variables related to the outcome under study

4. For time-varying factors that may have been affected by the comparator drug prior to switching, consider using an interaction term of these factors by the incident/prevalent new-user status.

5. For positivity purposes, verify for each exposure set that the time-conditional propensity score of the exposed subject lies within the range of the time-conditional propensity scores of the members of the corresponding exposure set, else eliminate the exposure set.

6. Starting chronologically with the first subject using the study drug, select the subject (or subjects if more than 1:1 matching) from the exposure set with the closest time-conditional propensity score. Subjects selected as comparators may or may not be eligible for subsequent exposure sets (see Discussion). 